\title{
Prospects and Challenges of Workplace Diversity in Modern Day Organizations: A Critical Review
}

\author{
Helen Eboh CLETUS, \\ Asia Graduate School of Business, Unitar International University, Selangor D.E., Malaysia \\ heleneboh@yahoo.com \\ Nor Asiah MAHMOOD, \\ Asia Graduate School of Business, Unitar International University, Selangor D.E., Malaysia \\ norasiah@unitar.my \\ Abubakar UMAR, \\ Malaysian Institute of Human Resource Management, Selangor D.E., Malaysia \\ abubakarumarr@gmail.com \\ Ahmed Doko IBRAHIM, \\ Department of Quantity Surveying, Ahmadu Bello University, Kaduna State, Nigeria \\ adibrahim2@yahoo.com
}

\begin{abstract}
Workplace diversity (WPD) is a holistic concept that denotes the differences that exist between people working within an organisation. It describes the complex physical, sociological or psychological attributes such as gender, ethnicity, sexual orientation, religious or political beliefs that define an individual or group. Therefore, WPD transcends the recruitment, representation or preferential treatment of people within an organisation. The complexity of WPD has become one of the most challenging issues currently of critical importance in business and organisational management. Therefore, the paper presents the critical issues currently impacting on WPD in modern day organisations. It identified and highlighted the various prospects and challenges of WPD. The results revealed that diversity in the workplace enhances the critical thinking, problemsolving, and employee professional skills. Furthermore, it enables organisations to attract talent, improve corporate attractiveness, productivity. However, it is currently hampered by the hostility, disrespect and discrimination people with diverse background encounter in the workplace. Such behavioural attitudes as ascribed to prejudices and biases towards people with varied lifestyle choices, ethnic and cultural differences, disabilities, and generational gaps. These factors can stifle morale, teamwork, profitability and the attractiveness of the organisation. Consequently, modern day organisations need to address the causes of these issues to exploit the benefits of WPD. These can be addressed strategies that foster an empowerment culture, build communication and team spirit with organisations. These efforts will promote acceptance, productivity, and profits in future organisations.
\end{abstract}

Keywords: Workplace, Diversity, Organizations, Demography, Personal Differences. 


\section{Introduction}

Over the years, the diversity of the global populations has been transformed by socioeconomic, geopolitical, environmental and technological factors. These dynamics have consequently changed the compositions of many societies and influenced the way human beings interact, work or cohabit with one another. As a result, many organisations are exploring strategies to positively exploit the growing diversity to remain and or gain a competitive advantage. According to the Pew Research Centre, the existence of a single racial or ethnic majority in the United States of America (USA) will cease to exist by 2055 . Other studies estimate that over 20 million people currently actively employed in the USA were born abroad. As a result, it is estimated that the USA and many nations worldwide will become pluralistic shortly [1]. This paradigm shift in the global demographic mix will simultaneously present challenges and prospects in the near future.

As a result, numerous organisations are actively integrating diversity into the recruitment processes to ensure the best talents are hired from around the globe. Many analysis thus project the future workplaces will be comprised of diverse groups of people drawn from various parts of the world [2]. Furthermore, it is predicted that the active movement of people from various will consist of all works of life and backgrounds will transform view sociocultural dimensions. However, the rapid diversification will challenge people's perceptions of the demographic factors of race, language, ethnicity, gender, age, and physical disability in the global workplace [3]. Other factors such as sexual orientation, socioeconomic status, religious and political ideologies will become prominent in the future. Given the challenges these factors will pose, many organisations have elected to adopt and prioritise workplace diversity. According to Dike [4], many organisations currently address the adoption of diversity to foster creativity and openness in the workplace. Similarly Christian et al., [5], opined that the adoption of workplace diversity has become an important corporate strategy to improve organisational management, competitive advantage and maximise profits. According to Amaliyah [6], modern organisations now recognise the critical need to adopt, invest and implement diversity as a corporate strategy for talent management. The findings also reveal that diversity can be a crucial link between corporate mission or vision and the overall performance [6].

Despite the growing significance of diversity, there is a dearth of knowledge on its impact on the future of the workplace, societies and the global economy. To adequately understand the concept, it is important to explore the various definitions of definitions currently propounded in literature. According 
to Patrick and Kumar [2], diversity can be defined as a collection of conscious practices that require comprehensive understanding and appreciation of humanity, cultures and the natural environment. It also involves a commitment to mutual respect for qualities and experiences of individuals who have different attributes. In addition, diversity studies aim to shed light on the challenges individual, cultural, and institutionalised discrimination creates in the world today. Most importantly, diversity studies aim to highlight the disadvantages discrimination presents for others and build alliances that enable people of diverse attributes to work together [2]. The study by Amaliyah [6], defines the diversity as the peculiar differences of people arising from physical, social, or psychological dimensions in the society. In a later study O'Donohue [7], described the concept as the process of recognising, understanding and accepting the differences of individuals regardless of age, class, ethnicity, gender, physical ability, race, sexual orientation, or religious inclinations. Previous studies have defined diversity as the process of acknowledging, understanding or appreciating differences such as age, class, race, ethnicity, gender, and disabilities atypical people [8].

As highlighted, the various definitions of diversity outline the concept as one that encompasses acceptance and respect between different people. Furthermore, it denotes a mutual understanding of the unique attributes inherent in individuals. However, it also eschews acceptance of the peculiar differences such as race, ethnicity, gender, sexual orientation of people. Hence, the concept promotes tolerance of peoples notwithstanding economic status, age, and disability, religious or socio-political beliefs. The exploration of individual differences in a safe, positive, and nurturing environment along with understanding, tolerance and embracing diversity is critical to the future of humanity. The exploration and exploitation of the rich dimensions of diversity are critical to how humans will interact, work or cohabit with one another in the future. Furthermore, workplace diversity has become a recurring feature in global discussions on the future or work and international business. This is has given rise to the concept of workplace diversity and its role in the organisational management of firms around the globe. So what is workplace diversity?

Workplace diversity (WPD) is a concept denotes the differences between people working in or for an organisation (Amaliyah, 2015). This can also mean the multiplicity of differences amongst individuals and their beliefs about they identify themselves as well as how others distinguish them in that organisation [9]. The concept describes the physical, sociological or psychological attributes that define an individual or group. These characteristics typically encompass demographics such as gender, ethnicity, education, socioeconomic background 
and also the individual's sexual orientation, geographic location, religious or political beliefs [10]. However, diversity in the workplace goes beyond the recruitment, representation or preferential treatment of people with specific birth characteristics in an organisation. The authors opine WPD is the process whereby employers avail potential talent or current employees with equal opportunities to thrive regardless of race, ethnicity or any other distinguishing factors [2].

With the evolving global business environment, companies are currently adapting their policies and strategies to appeal to future talent effectively. As a result, workplace diversity continues to gain prominence among companies who continuously seek global expansion, and maximisation of profits amidst growing competition for scarce talent, resources and market share. Analysts opine that, in the coming years, companies and organisations will need to devise effective strategies and increase investments in diversity programmes to remain globally competitive. Others predict that the firms with best WPD strategies will attract the largest pool of talent, maximise recruitment, lower operational costs amongst other benefits. Empirical research has shown that the profit margins of companies with diverse teams of employees are $35 \%$ higher than homogeneous firms [11].

However, WPD presents challenges to many organisations. The recruitment of people of diverse backgrounds, cultures and characteristics can present problems in the workplace. The importance, complexity, and holistic nature of the concept mean organisations are required to address the problems that may arise from hiring talents or operations in regions with diverse peoples and cultures. According to Robinson et al., [12], the constantly changing dynamics of global business requires companies to embrace inclusion in the face of diversity. This will enable the firms to address not only WPD but also increase productivity, profit maximisation, and competitive advantage in the ever increasing competitive climate of global business and organisational management. Based on the preceding, it is evident that for firms, companies or organisations to effectively compete and succeed in the current global economic climate, there is an urgent need for identify, examine, and highlight the merits and demerits workplace diversity. To the best of the authors' knowledge, there are limited studies highlighting the prospects and challenges of workplace diversity (WPD) in modern-day organisations in the scientific literature. Therefore, this paper seeks to identify, examine, and highlight these outlined issues to addressing the gap in knowledge in the subject area. It is envisaged that the findings will benefit managers and organisations seeking to improve workplace diversity, promote productivity and maximise profits in the increasingly competitive climate of global business and organisational management. 


\section{Prospects and Benefits of Workplace Diversity}

The concept of diversity is critical to the success of companies and organisations in today's global marketplace where people of different cultures that interact and conduct business. The potential benefits of diversity can result in increased creativity, productivity, and profit maximisation. Other potential benefits include the infusion of novel ideas, attitudes, and skills. According to Green et al., [3], diversity is critical to the ability for businesses to attract and retain its important customers, employees, and partners. As a result, businesses are required to explore and exploit the diversity of the people critical to their success. This view is corroborated by Ashe and Nazroo [13], whose study demonstrates that the most important asset of an organisation is its people. Hence, organisations need to identify and exploit the different races, genders, abilities, ages and cultural differences of its employees and clients around the globe to ensure success. This will potentially result in numerous benefits as enumerated below.

\subsection{Critical Thinking and Problem Solving Skills}

The existence of workplace diversity can improve critical thinking and problem-solving skills in organisations. Therefore, employees from different backgrounds and experiences can present a variety of perspectives to the workplace [14]. Consequently, diverse employees in a firm can provoke critical thinking that provides not only alternative solutions and approaches to problemsolving but the needed edge to succeed in a competitive business environment. Furthermore, the diversity permits sharing of personal views, experiences and approaches to the culture of the current workplace. These dynamics can help nurture local talent and improve the culture of work and doing business in an organisation. Furthermore April and Cheah [14], revealed that even the biases, prejudices or perceptions of people of diverse cultures could provide lessons in conflict resolution in the workplace if appropriated managed and channelled resolutely. The authors state when the strengths and visions of every individual are harnessed, productivity and better results can be achieved in the long run. The diverse composition of teams in organisations can determine its potential for success. This assertion is mirrored by Walia and Malik [15], whose study revealed that workplace diversity provides employees with varied capabilities that can ensure strategic planning and execution of management. These skills according to the authors are essential components for enhanced 
communication, collaboration, and conflict resolution in the workplace [15]. Lastly, the study showed that workplace diversity could provide multivariable perspectives and numerous alternative viewpoints in an organisation.

\subsection{Employee Growth and Development}

Workplace diversity has the potential stimulate employee growth and personal development in organisations. In the light of growing diversity in business and workplaces around the globe, employees can experience growth and personal development. The exposure of employees in an environment of varied culture, opinions and ideas can stimulate personal growth and skills development. Hence, individuals with diverse characteristics can provide a platform for creating and testing innovative ideas [14]. This view is also shared by [1] who states that diversity enhances the approach to troubleshooting and problem-solving in organisations. As a result of the multiple perspectives and approaches, employees can effectively address the various tasks or issues, decipher problems and create innovative solutions in the workplace. Also, the existence of people of various backgrounds in companies and organisations improves the ability of its employees to acclimatise to different circumstances. This can also enhance the employee's ability to work around the differences in personality, cultures and background in the working environment. The fundamental notions of ethnocentric are ultimately elevated to the fore, confronted and dispensed when workers work with colleagues with varied cultures and styles in a diverse workplace [4].

\subsection{Unification of diverse strengths}

Diversity presents prospects for companies and organisations to exploit the diversity of its employees to achieve its workplace goals, tasks, and long-term plans. Furthermore, Dhuppar [10], revealed that diversity has the potential to unite the collective strengths and inherent weakness of employees in an organisation to its advantage. According to the findings of the study, the different skills and strengths of employees of various backgrounds can be harnessed for better performance and productivity. For example, this can be achieved by merging the various technical, management, and sales strengths of various employees. Similarly [1], revealed that cross-cultural understanding could stimulate better working environment which can go a long way in dispelling stereotypes, prejudices and misconceptions about people of diverse cultures. Hence, workplace diversity can help unite and strengthen the bonds between people of diverse backgrounds [16]. Ultimately, this scenario improves 
not only working groups but also the climate for the effective organisational management and the achievement of the organisation's goals $[3,8]$.

\subsection{Promotes Corporate Attractiveness}

Workplace diversity can increase the attractiveness of companies and organisations to potential business partners, collaborators, and the society at large. Over the years, diversity studies in the workplace have shown that firms that hire and retain employees of diverse backgrounds received positive attention from the media, society and governments worldwide. According to Robinson and Dechant [17], companies that promote workplace diversity and corporate inclusion can bolster their attractiveness to future employees and clients. Also, the added publicity can catapult companies to the apex of the talent pool. In the era of scarcity of talent and skilled employees, attracting the best talent can further enhance the profitability, survival and future of companies. Diversity increases the prospects of companies to explore novel ideas and styles which can appeal to the exploratory minded group of employees particularly millennials and generation $Y$. The study further stated employee focused organisations can improve their attractiveness to the employees present and future, clients and the society by adopting workplace diversity [17]. Similarly, the McKinsey report revealed that workplace diversity enhances employee satisfaction and conflict-free relationships between employees. This improves not only collaboration and loyalty but also the attractiveness of the firm to potential employees, business partners and the society at large [11]. In the long run, a people-centric approach also assists companies and organisations to understand the target demographics better and improve product delivery, maximise profits and customer satisfaction [9].

\subsection{Fosters Innovation}

The success of companies and businesses is hinged on their ability to innovate, adapt, and evolve along with the changing trends in the business climate. With the growing diversity around the globe, these organisations must tap into the diverse talent pool to remain competitive, relevant and best practices. This presents significant opportunities to innovate, as employees of different backgrounds often arrive with diverse skill sets that nurture innovative ideas and critical thinking. This view is aptly buttressed in the maxim, "diversity breeds innovation and innovation breeds" [18]. Furthermore, multi-lingual employees or workers from variable sociocultural backgrounds are critical to the 
expansion of companies into international, national, regional and local markets $[9,19]$. As such diversity, can strengthen customer orientation and improve target marking and advertising [11]. It is believed that the multilingualism and sociocultural skills can foster innovation, profit maximisation, and workplace attractiveness [9]. Furthermore, the diverse perspectives, experiences, culture, gender and age of employees can present a fresh outlook toward to the workplace $[8,18]$.

According to McKinsey and Company [11], innovation and creativity are catalysed by workplace diversity. As a result, there is significant multiplicity in the approach to problem-solving, ideological perspectives, innovative ideas, and decision making $[11,18,20]$. Furthermore, research in the academia has demonstrated that diverse groups often outperform so-called experts working in related disciplines. In a separate study, CTI (Centre for Talent Innovation) verified the correlation between improved decision making and sociocultural diversity. The study further highlighted that inclusive leadership could help companies maximise the benefits of diversity and avoid the pitfalls of homogeneous workplaces which reportedly stifles innovation [21, 22]. Lastly, investments in workplace diversity help companies and organisations maximise profitability and nurture innovation. Over time, the financial earnings of such companies are not only greater than the national averages, but it competitively differentiates them from others in the market [2].

\section{Challenges of Workplace Diversity}

Diversity is a holistic concept currently undergoing socio-cultural metamorphosis around the globe. Also, the complex nature of the concept makes it one of the most challenging issues currently facing modern day organisations. Given this, workplace diversity has become a recurring issue in debates in business and organisational management circles [20, 23, 24]. As a result, analysts predict that diversity and its importance in the global international business climate will soar geometrically in the future due to growing increase population demographics [11]. Furthermore, its emerging prominence requires firms to adopt and or adapt strategies to address the challenges currently posed by diversity in the workplace[15, 17]. Therefore, it is essential for organisations to focus on diversity and explore holistic inclusion because diversity has the potential of yielding greater productivity and competitive advantages [12]. Therefore, it is important to highlight the various forms of diversity and the potential challenges each poses in the workplace. 


\subsection{Gender and Lifestyle Choices}

In many organisations worldwide, the gender and lifestyle choices of its employees have become an important issue of debate. This is based on the premise that the cognitive biases, behaviour and attitudes of employees in the workplace can influence affect decision making and invariable problems [11]. However, empirical studies have elucidated that these choices do not impact on the workplace performance. Nonetheless, many employers and their organisations expend significant efforts to highlight and extend workplace benefits to people with alternative lifestyles [10]. For example, the business world acknowledges that members of the LGBT (lesbian, gay, bisexual and transgender) community significantly suffer hostility, disrespect and discrimination from colleagues [21]. Behavioural attitudes as such can result in several challenges not limited to an uncomfortable atmosphere and poor productivity in the workplace $[3,10,22]$. Similarly, gender is a key focal point in many organisations due to its relation to human diversity, capital, and quality of life [25]. As a result, gender-related issues such as pay disparities, promotions and decision making power amongst male, female and transgender in organisations remains a thorny issue. As such antiquated notions that project women as the weaker sex and hence fit for only temporary or part-time or low responsibility jobs persist to date [26]. Furthermore, the common belief is that women are predisposed to marriage, and childbearing hence will quit even the pinnacle of their careers to focus on family. Furthermore, women are considered physically, mentally, and emotionally incapable men [3].

\subsection{Ethnic and Cultural Differences}

Many factors such as language, religion, race, sexual orientation, gender, age, and ethnicity account for the ethnic and cultural differences of human beings. As a result, the factors can result in hostility, discrimination or disrespect in the workplace environment. However, since today's workplace comprises of individuals from all part of the world, diversity and the challenges it poses are considered crucial to organisations. The diversity of working populations has created an atmosphere of multiracial and multicultural dynamism in many organisations $[9,11]$. As a result, it is expected that some individuals will not only harbour unfair prejudices but also project their inherent biases, anger and frustrations to others considered different in the organisation. Also, such behavioural tendencies result in stereotyping of people of diverse cultures, 
ethnicity or religion [10]. In the end, such attitudes can result in poor cohesion, team bonding, and create other problems such bullying, scapegoating or harassment in the workplace. The term harassment is defined as the extreme conducts, actions or utterances against an individual that create an abusive, hostile or intimidating environment for work. In practice, harassment occurs because of the race or colour, religion, or nationality of an employee. Other factors can include disability genetic information or affiliation to selected social groups. According to Amaliyah [6], these issues significantly impact on organisations in a diversified work environment cannot be tolerated or permitted to thrive. Therefore, organisations need to carefully consider these challenges ethnic and sociocultural differences can have on the workplace.

\subsection{Physical or Mental Disabilities}

The term disability describes the state in which a physical or mental injury afflicts an individual. In practice, such impairments may have resulted in considerable and long-term adverse effects on the individual's capacity to perform customary day-to-day activities. Although, disabilities do not entirely prevent an individual from the capacity to perform workplace tasks or duties but can present challenges or limitations [3]. In selected cases, workers with disabilities who are mentally or physically handicapped face harassment, discrimination or ridicule from impervious colleagues. As a result, employers can sometime side-step employing individuals with mental, physical or other disabilities. This is based on misconceptions that people with disabilities are "slow" or incapable of comprehending or completing workplace goals, tasks or demands. Such prejudices extend to the disregard for the needs and rights of disabled workers such as ramps or special needs equipment (Green et al., 2002) which some employers consider expensive and unnecessary. Furthermore, this perceived disregard for the rights of people with disabilities can trigger hostilities, discrimination and bullying in the workplace. In the long run, the workplace becomes hostile and unfriendly resulting in low morale, productivity and attractiveness of the organisation. Therefore, the physical or mental disabilities of present and future employees of organisations need to be addressed in the workplace $[4,21]$.

\subsection{Workplace Communication}


Communication is an important tool that creates and maintains cohesion in the workplace. Also, fluid communication is crucial to the attainment of the organisations' goals, tasks and management. Therefore, the peculiarities of individuals in the workplace are vital for the realisation of these objectives. Since each person brings his or her individuality to the workplace, the contributions of each individual can enhance productivity, quality of work, relationships, and even loyalty of a workplace [4]. However, poor communication can result in confusion, lack of teamwork, and low morale in the workplace. Similarly, lack of effective communication of the firm's objectives can result in missed deadlines, incorrect goal assumptions and ultimately conflicts in the workplace. For example, hiring workers with poor language proficiency can affect hinder collaboration, communication and productivity among team members [2]. For example, if an American company employs people whose lingua franca is not English, other employees and managers will experience communication problems. The multicultural diversity can result in misunderstanding and decrease in productivity [10]. As a result, firms and organisations need to identify, examine and address the problems workplace diversity can present in the workplace.

\subsection{Generation Gaps}

The difference in generation gaps is one of the biggest challenges of workplace diversity. The resultant effect is the lack of cohesion caused in part by lack of communication between the old and newer generations. This creates problems in the workplace particularly in times when strategic changes are required for the advancement of organisations. In the light of this, generations that are far apart experience problems during the adjustment phase which is often viewed regarding generational differences but a change in the career cycle [27]. In theory, the various generational gaps that exist are; traditionals, baby boomers, generation $\mathrm{X}$, millennials, and the iGeneration.

Born before 1945, the class of traditionals were raised in times of significant economic hardships which moulded this group into disciplined and self-sacrificing individuals. Nowadays, most are retired from active service in the workplace. However, the baby boomers who were born from 1945 to 1964, were raised during economic prosperity, suburban affluence, and sturdy extended families steered by strong stay-at-home mothers. According to Birkman [27], this generation, aged 52 years on average, presently dominates the workplace in the US. 
Next, the generation X, born between 1965 and 1980, were raised as "latchkey" kids in the turbulent era of divorce and working mothers. According to the author, this generation is characterised by independent, resilient, adaptable individuals who take pride in the maxim "I do not need someone looking over my shoulder". As a result, this generation is frequently ignored in favour of the preceding and succeeding generations on their either side [27].

The millennials, on the other hand, were born between 1981 and 1999. This generation was born in what can be best described as the most child-centric time in human history. Hence, children born in this era are not only overly confident and seemingly cocky but are also showered with great attention amidst high expectations from their parents. This group is also known as the generation $\mathrm{Y}$, and are the largest generation in the workforce of the world still considered as growing.

Lastly, the iGeneration comprises of children born from 2000. Analysts predict this generation be exceptional employees in the future due to their capacity to pick up new skills and ideas such as information technology (IT) required for employment in many business organisations [28]. This group is also known as Generation Z [27]. In large organisations, the ages of employees' age can range from teenagers to senior citizens. These generation gaps present workplace issues as the differences can trigger "cliques" and create divisions within the company. Bridging this chasm can sometimes become a thorny issue between multiple generations amidst efforts to create teamwork [10].

\section{Way Forward for Workplace Diversity}

Despite the outlined prospects diversity several challenges persist in the modern-day organisation. The natural obstacles to embracing and implementing diversity in an organisation require organisations to stand up and take notice or risk isolation in the increasingly competitive world of local and international business. It is no longer just the question is how can issues of workplace diversity be resolved, but what steps, programmes and strategies are required in the present and near future. Therefore, it is imperative that companies chart a sustainable course for addressing the issues raised to tap into scarce talent pools, increase efficiency, increase market share and maximise future profits. The problems and issues raised by workplace diversity can be resolved by looking at the following steps.

\subsection{Understand Discrimination}


Given its importance, employees, supervisors and managers must be required to understand the company's policies, guidelines and legislation on workplace diversity. This will enable all stakeholders to identify and curtail any acts of hostility, harassment or discrimination of people of diverse cultures within the organisation. Furthermore, understanding discrimination and the company's policies on the matter. This will not only promote effective communication but also reduce the incidence of conflicts and behavioural issues in the workplace [29]. Furthermore, the effective communication and understanding can assist companies to devise and enforce strict yet acceptable penalties for parties that partake in discriminating other colleagues. Lastly, Green et al., [3] affirm that knowledge of workplace legislation can guard against discriminatory tendencies of employers in the decision-making process and prevent prejudices that either unduly favourable or reprimand employees based on colour, religion, age, gender, and race.

\subsection{Determine Individual's Preconceptions}

It is imperative for managers in the workplace to identify and address any misconceptions and biases. This can promote better conflict resolution and prevent inconsistent decision making as regards to matters of diversity in the workplace. Furthermore, understanding the biases between employees can assist managers thoroughly examine and cultivate practical solutions for managing employees particularly in social and multicultural and diverse organisations [26]. According to Green et al., [3] organisations can address diversity issues in the workplace by outlining the bias factors held by managers and employees. The study reveals that this approach which has been successfully applied at the University of Florida mandates employers to openly highlight such biases in the workplace [3].

\subsection{Diversity Training Programmes}

Various studies have shown that diversity has a positive impact on many key aspects of organisational performance and workplace management. Accordingly, to the McKinsey report, programmes designed to address workplace diversity enhance the competitive advantage of companies in their search for talent [11]. Similarly, the CTI LGBT report revealed that diversity efforts aimed at target group's result in the discovery of excellent talent [21]. The study also observed that on average LGBT (lesbian, gay, bisexual, and transgender) workers 
are more likely to have high skills or advanced degrees [21, 22]. Hence, organisations that strive to excel amidst the changing demographic transitions are required to invest in diversity training sessions. Also, these efforts need to not only reinforce company policies but also inspire appropriate behaviour towards people from diverse cultures. It is envisaged that such training and professional programmes will equip stakeholders with tools and strategies to address challenges that may arise among the "melting pot" of employees [3]. Lastly, diversity training should be adopted as a strategic tool to shape the organisation's diversity policy. This approach will help alter the leadership teams or staff composition required to interrupt old habits and routines that favour or discriminate against people of diverse cultures in the organisation [11].

\subsection{Empowerment Culture}

Managers in organisations create an empowerment culture to address the problems of diversity in the workplace. This can be achieved by infusing an atmosphere that welcomes and empowers employees with diverse skills to work in teams. It is also important to equally contribute to training and awarding without gender discernment. Managers can be very effective in helping to establish morale and encourage employees to do their best [3]. The process should also involve all employees from the process of formulation and execution. Lastly, diversity initiatives in the workplace should help to defend against resistance to change and afford them a sense of belonging [30].

\subsection{Workplace Orientation}

Organizations orientate fresh employees to familiarise themselves with the operations and the current workforce. With an appropriate definition of company policies, issues or concerns will be addressed. Also, queries will be answered, as well as the short and long-term goals can be discussed. This will be a good orientation to welcoming the newcomers and make them feel home. Therefore, organisations can establish customizable employee satisfaction survey that provides comprehensive reporting. The results can be used to build and implement successful diversity in the workplace policies [30].

\subsection{Define Job Descriptions}


It is crucial for organisations to highlight and describe the skills required for jobs. This will enable the organisations to hire the best personnel from the increasingly limited pool of talent. Therefore, managers rapidly determine an individual's ability to handle the potential challenges of a diverse workforce during the interview process. Furthermore, this will allow managers select the most aspiring employees from the pool of candidates who value and understand the importance of workplace diversity and ultimately help manage other candidates who do not [30]. According to Speroni et al., [26], such measures can help companies create policies and guidelines that clearly outline the inclusive nature of recruitment. Also, this will enable the organisation present a blueprint for compensation, benefits, professional development, and social programs beneficial to its employees from the outset. Lastly, such policies serve to highlight that the organisation maintains a top-down culture of inclusivity; and will not tolerate harassment and discrimination as well as the consequences for violating such policies [26].

\subsection{Communication and Team Building}

The twin tasks of effective communication and team building are crucial exercises in any modern-day organisation. The implementation of these processes enables employees to flourish, and it brings about trust amongst them regardless of the level of diversity. For example of team building activities such as strategic games, sharing stories and experience, role play and much more can help employees bond and communicate more effectively. It helps others "break the ice" by easing their nervousness. According to the study by Podsiadlowski et al., [30], such activities create and strengthen bonds in the workplace. Ultimately, it improves effective communication and creates avenues for appreciation of the workplace and co-workers alike. Similarly, the free flow of communication is an additional important factor even the sturdiest and competent organisations require for long-term survival. According to the authors, it all about sharing ideas, concerns, queries and solutions to ensure a healthy environment. 


\section{Conclusions}

The paper examined and highlighted the critical issues impacting on the concept of workplace diversity in modern-day organisations around the world. As a result, the authors identified, examined and highlighted the various prospects and challenges of diversity in the workplace. This is based on the premise that modern day firms, companies or organisations need to adapt to effectively compete and succeed in the face of growing diversity and current global business climate. According to the finds, diversity promotes critical thinking and problem-solving skills, employee growth and development, unification of diverse strengths, corporate attractiveness, and innovative ideas and skills in the workplace. However, these benefits are currently plagued by several factors including but not limited to the following. Firstly, the gender and lifestyle choices of people, ethnic and cultural differences, physical or mental disabilities, workplace communication, and generation gaps can result in problems in the workplace. As a result, people in these groups face hostility, disrespect and discrimination from other colleagues. According to the findings, such behavioural attitudes result in an uncomfortable atmosphere and poor productivity in the workplace. Also, these challenges can dampen morale, confusion, affect teamwork, and lower the attractiveness of the organisation. Consequently, modern day organisations need to address the causes of these issues to benefit from the long-term benefits of diversity. To this effect, this paper proposes a way forward for improving diversity in the workplace. Firstly, organisations need to understand better discrimination through diversity training and orientation programmes that will optimistically determine individual's preconceptions in the workplace. It is envisioned that this will foster an empowerment culture, define job descriptions, and lastly build communication and team spirit among an organisations employees. Based on these presentations, the authors envisage that the findings will benefit future employees, managers and organisations adapt to the realities of workplace diversity. Also, the authors hope the findings to improve workplace diversity, promote productivity and maximise profits in the increasingly competitive climate of global business and organisational management. 


\section{References}

[1] Emma, L. Advantages and Disadvantages of Diversity in Workplace. Workplace Diversity 201806 June 2018]; Available from: https://bit.ly/1jdaWY0.

[2] Patrick, H.A. and V.R. Kumar. (2012). Managing workplace diversity: Issues and challenges. Sage Open, 2(2): 2158244012444615.

[3] Green, K.A., M. López, A. Wysocki, and K. Kepner. (2002). Diversity in the workplace: Benefits, challenges, and the required managerial tools. University of Florida, 1(4).

[4] Dike, P. (2013). The impact of workplace diversity on organisations. Department of International Business, PhD: 59.

[5] Christian, J., L.W. Porter, and G. Moffitt. (2006). Workplace diversity and group relations: An overview. Group Processes \& Intergroup Relations, 9(4): 459-466.

[6] Amaliyah, A. (2015). The Importance of Workplace Diversity Management. International Journal of Sciences: Basic and Applied Research, 17(2): 175-182.

[7] O'Donohue, M. (2016). Using a Knowledge Management Approach to Support Effective Succession Planning in the Civil Service. Computer Sciences, Masters Dissertation (MSc).

[8] Esty, K.C., R. Griffin, and M.S. Hirsch. (1995). Workplace diversity.

[9] PeopleScout. Managing Diversity in the Workplace. Diversity 2018 [cited 2018 6th June 2018]; Available from: https://bit.ly/2rqbEYU.

[10] Dhuppar, S. (2015). Managing Workplace Diversity Challenges and Strategies. International Journal of Research, 2(3): 346-351.

[11] McKinsey and Company. Why diversity matters. McKinsey Insights 2018 [cited 2018 6th June]; Available from: https://mck.co/2lvVXjd.

[12] Robinson, D., H. Fenwick, and W. Lip. (2002). Experiencing diversity in the workplace Institute of Food and Agricultural Science Extension: 11-13.

[13] Ashe, S. and J. Nazroo. (2017). Equality, diversity and racism in the workplace: A qualitative analysis of the 2015 race at work survey.

[14] April, S. and B.L. Cheah. Why workplace diversity is important for every organisation. Career Guide 2013 6th June 2018]; Available from: https://bit.ly/2JweWEY.

[15] Walia, M.S. and R. Malik. (2015). Workforce Diversity Management: Essence of Modern Organisations. Editorial Board.

[16] Amin, A. (2002). Ethnicity and the multicultural city: living with diversity. Environment and planning A, 34(6): 959-980.

[17] Robinson, G. and K. Dechant. (1997). Building a business case for diversity. The academy of management executive, 11(3): 21-31.

[18] Hospers, G.-J. (2003). Creative cities: Breeding places in the knowledge economy. Knowledge, Technology \& Policy, 16(3): 143-162.

[19] Deari, H., V. Kimmel, and P. Lopez. (2008). Effects of cultural differences in international business and price negotiation.

[20] Zahra, S.A. and S. Nambisan. (2011). Entrepreneurship in global innovation ecosystems. AMS review, 1(1): 4.

[21] Hewlett, S.A., T. Sears, K. Sumberg, and C. Fargnoli. (2013). The Power of "Out" 2.0: LGBT in the Workplace. Center for Talent Innovation: 30.

[22] Hewlett, S.A., M. Marshall, L. Sherbin, and T. Gonsalves. (2013). Innovation, diversity and market growth. 
[23] Guillén, M.F. (2000). Diversity in globalization. Organizational change in Argentina, South Korea and Spain: Discussants: Evelyne Huber and Michael Mosher, Paper, 6: 1-41.

[24] Rowntree, L., M. Lewis, M. Price, and W. Wyckoff. (2015). Diversity amid globalization.

[25] Mahmood, N.A., J. Uli, and S.M. Shah. (2017). Gender and Job Category as Moderator Effects on Relationship between Human Capital and Quality of Life (QOL).

[26] Speroni, K.G., T. Fitch, E. Dawson, L. Dugan, and M. Atherton. (2016). Managing diversity and equality in the workplace. Nurse Education in Practice, 3(1): 1-14.

[27] Birkman, I. How Generational Differences Impact Organizations \& Teams. eBook Series 2016 6th June, 2018]; Available from: https://bit.ly/2JldYIn.

[28] Nyakuma, D.D., A.J.K. Shittu, H. Ojobo, and T.J.-P. Ivase. (2016). Challenges Of Adopting Information And Com-munications Technology By Small And Medium Enterprises In Nigeria. Journal of Multidisciplinary Engineering Science and Technology, 3(1): 3766-3776.

[29] Ryan, T. (2016). 5 Strategies for Dealing With Diversity in the Workplace. . Hearst Newspapers

[30] Podsiadlowski, A., D. Gröschke, M. Kogler, C. Springer, and K. Van Der Zee. (2013). Managing a culturally diverse workforce: Diversity perspectives in organizations. International Journal of Intercultural Relations, 37(2): 159-175. 